\title{
Linear and nonlinear light beam propagation in chiral nematic liquid crystal waveguides
}

\author{
Urszula A. Laudyn ${ }^{1}$, Michał Kwaśny ${ }^{1}$, Pawel S. Jung ${ }^{1}$, Marek Trippenbach ${ }^{2}$, Gaetano Assanto, ${ }^{3,4}$ and \\ Mirosław A. Karpierz ${ }^{1}$ \\ ${ }^{1}$ Faculty of Physics, Warsaw University of Technology, Koszykowa 75, 00-662 Warszawa, \\ ${ }^{2}$ Faculty of Physics, Faculty of Physics, University of Warsaw - ul. Pasteura 7, PL-00-681 Warszawa, Poland \\ ${ }^{3}$ Optics Lab, Department of Physics, Tampere University of Technology, 33101 Tampere, Finland \\ ${ }^{4}$ NooEL-Nonlinear Optics and OptoElectronics Lab, University of Rome "Roma Tre", 00146 Rome, Italy
}

Received March 17, 2016; accepted March 30, 2016; published March 31, 2016

\begin{abstract}
We employ a thick layer of chiral nematic liquid crystals to demonstrate the evolution of a one-dimensional (1D) higher-order guided mode into a beam self-confined in both transverse dimensions at various wavelengths. We also report the experimental observation of higher-order modes guided by soliton-induced waveguides in chiral nematic liquid crystals.
\end{abstract}

Nematic liquid crystals (NLCs) are one of prime examples of nonlinear, reorientational soft matter which, owing to nonlocality, support the generation of stable spatial solitons also known as nematicons [1-3]. Nematicons were demonstrated and investigated in several NLCs geometries including planar, homeotropic, twisted and chiral [2-3]. They can be exploited in reconfigurable optical circuits created by light alone, where all-optical switching/processing can be achieved by acting on power and/or the interaction of solitons and beams [4-5]. An attractive property of these solitoninduced waveguides is their ability to guide weak signals at different wavelengths [6-9]. Recently, higher order modes have been reported experimentally, guided by fundamental solitons in NLCs with planar orientation [10].

In this Letter, we investigate chiral nematic liquid crystals (ChNLCs), i.e., a nonhomogeneous NLC configuration in which the mean direction of molecules varies across the cell thickness [11-15]. This arrangement results in helical structures with a defined pitch $\mathrm{p}$, defined as the distance along the helical axis after which molecular orientation has rotated by an angle $2 \pi$. In principle, light propagation and nematicon formation resemble those in pure NLCs with a planar texture $[2,12]$ : solitons can be observed over distances of a few $\mathrm{mm}$, they exist in a wide range of optical powers above a few $\mathrm{mW}$, their excitation depends on polarization, they can be redirected by external fields or structural defects. However, since the optical properties and response of NLCs are determined by their initial orientation, there are also several differences. At variance with other orientations, ChNLCs support nematicon creation and propagation independently in each separate layer of thickness p/2 [12-13]. The evolution of a higher order 1D guided mode into a 2D beam self-confined in both transverse dimensions as well as astigmatic solitons were demonstrated in ChNLCs $[13,15]$.

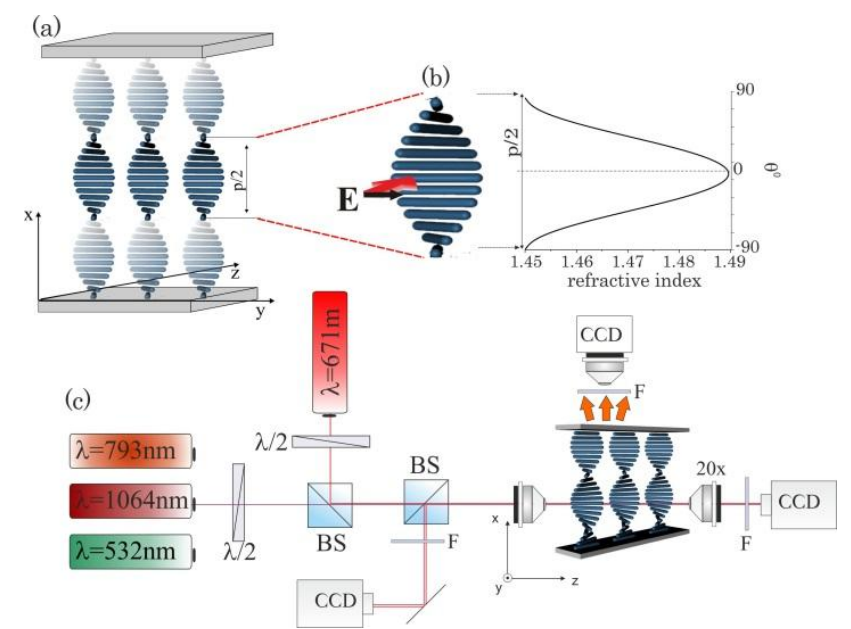

Fig. 1. Sample, experimental setup and coordinate system (a) Chiral structure of the ChNLC sample, with a sketch of orientation; (b) refractive index profile calculated for an extraordinary wave (transverse electric) in a half-pitch layer. (c) Experimental setup with various wavelength sources. BS is a beam-splitter, F a filter, CCD is a chargecoupled device camera.

The cell geometry is sketched in Fig. 1a. The chiral structure is sandwiched between two glass plates treated for planar anchoring along the same direction. A $y$ polarized light beam propagating along $z$ experiences a refractive index, continuously varying across $x$ from ordinary to extraordinary values. Such a graded-index structure, periodic with $\mathrm{p} / 2$ as directors $\mathbf{n}$ and $-\mathbf{n}$, which are equivalent (for nonpolar molecules), essentially forms a set of planar (1D) graded-index waveguides.

In the experiments we used a Gaussian $\mathrm{TEM}_{00}$ linearlypolarized beam from either a Nd:YAG laser operating at $\lambda=1064 \mathrm{~nm}$, or a Ti:sapphire laser at $\lambda=793 \mathrm{~nm}$, a frequency doubled Nd:YAG at $532 \mathrm{~nm}$ or a diode laser at 
$671 \mathrm{~nm}$. In dual beam experiments we employed $\lambda=1064 \mathrm{~nm}$ and $\lambda=671 \mathrm{~nm}$. The beam was gently focused to a waist of several micrometers at the input of the sample $(z=0)$ with a microscope objective. Another microscope objective at the cell exit and a CCD camera collected the light at the output (transmitted signal), while a second CCD camera allowed monitoring the evolution of the beam along $\mathrm{z}$ by imaging the light scattered out of the observation plane $y z$. The input beam position was controlled via the reflected signal using a third CCD camera. In this configuration light beams propagate in the $z$-direction, parallel to the glass plates. A nematicon can be generated by injecting a y-polarized beam with a wave vector parallel to $z$.

Due to the graded index profile of each layer, the injected focused beam can result in the excitation of a few modes. The number of excited modes in a layer depends on both the wavelength of the beam and its initial width, as well as its $x$-location within the layer thickness. To determine the approximate number of modes that can propagate in the layer, calculations were performed for light propagating in a planar waveguide with a graded refractive index distribution.

In this work we studied low-birefringent NLCs 1110 [16] with a chiral dopant at a weight concentration of about $1.78 \%$, resulting in a pitch of about $16 \mu \mathrm{m}$. Therefore, the thickness of each waveguide was set to $8 \mu \mathrm{m}$, half of the helical pitch. The distribution of the extraordinary-wave refractive index is a function of the orientation angle [2-3] and the eigenvalues $n_{\perp}$ and $n_{\|}$ associated to electric fields orthogonal and parallel to the molecular director (optic axis), respectively. Depending on wavelength, the refractive indices are: $n_{\|}=1.505$ and $n_{\perp}=1.459$ at $\lambda=532 \mathrm{~nm} ; n_{\|}=1.5$ and $n_{\perp}=1.454$ at $\lambda=793 \mathrm{~nm} ; n_{\|}=1.496$ and $n_{\perp}=1.4504$ at $\lambda=1064 \mathrm{~nm}$. Each half-pitch layer guide can support 5 transverse-electric (TE) modes at $532 \mathrm{~nm}$ and 3 TE modes at $793 \mathrm{~nm}$ and $1064 \mathrm{~nm}$. In order to excite a specific mode, the input beam needs to exhibit a transverse distribution close to it. As a result, launching a low-divergence beam in the centre of a layer (i.e., at half thickness), tends to excite the fundamental mode. Beams with a large divergence will also couple to higher order modes. Asymmetric modes, with a zero intensity in the middle of the layer, will be generated by beams launched with an offset in the layer. By adjusting the wavelength, the beam alignment and its width in the linear regime, we excited various modes, as shown in Fig. 2 at 532nm, 793nm and 1064nm, respectively. Varying the beam divergence (waist) we could launch the fundamental mode only or a combination of modes, their number dependent on the wavelength. The photographs were taken at the output of the cell (after $3 \mathrm{~mm}$ of propagation) showing a typical $\mathrm{n}$ - hump solution guided in $x z$ and diffracting in $y z$. Owing to one-dimensional spreading in the planar layer $y z$, the mode distribution appears uniform across the observation window.
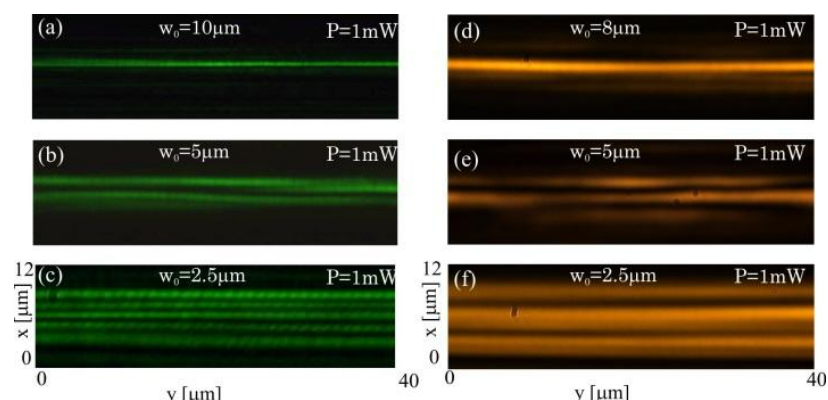

$\mathrm{y}[\mu \mathrm{m}]$

$\mathrm{y}[\mu \mathrm{m}]$
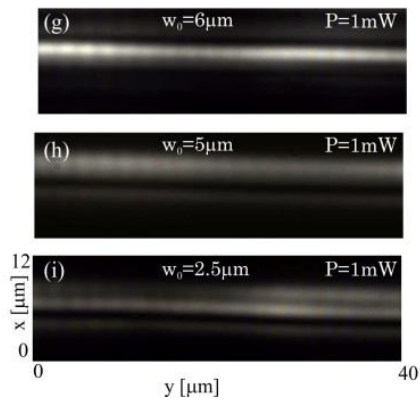

Fig. 2. Output profile of the fundamental (a, d, g) and higher (b-c, e-f, hi) order linear transverse-electric eigenmodes excited by a beam of power $\mathrm{P}=1 \mathrm{~mW}$ at wavelengths (a) $532 \mathrm{~nm}$; (b) $793 \mathrm{~nm}$; (c) $1064 \mathrm{~nm}$, respectively.

In the nonlinear regime, all-optical reorientation and high nonlocality of the response resulted in an extraordinary polarized wave distorting the molecular director distribution. Both the refractive index and the transverse electric field distribution became a function of the beam power. Increasing the input beam power led to self-focusing and nonlinear interaction between the modes. First, at low powers we observed a change in the oscillation period, as the nonlinearity has a greater impact on the propagation constant (or effective refractive index) than transverse distribution. Then, at higher excitations, the energy flow between the modes as well as their transverse distribution was observed to change. This led to the dominance of the mode with the highest propagation constant and the emergence of a spatial soliton, as visible in Fig. 3 at 532nm. The (fundamentalorder) bright spatial soliton is the nonlinear mode with the highest effective refractive index for a given power. 

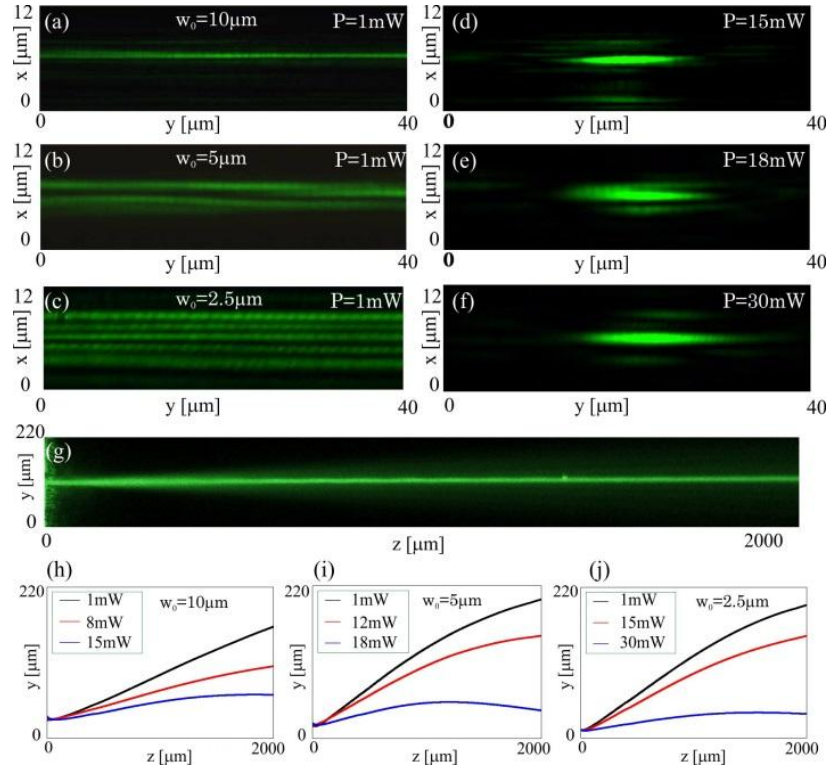

(j)

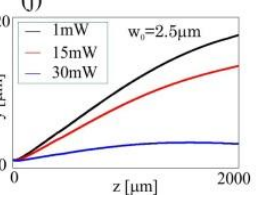

Fig. 3. Results at $\lambda=532 \mathrm{~nm}$. (a-f) Output profile of (left) the linear $1 \mathrm{st}$ order and higher order TE eigenmodes excited by a $\mathrm{P}=1 \mathrm{~mW}$ beam with various input beam waists (as marked) and (right) corresponding nonlinear mode for higher powers as specified. (g) Typical beam evolution at $\lambda=532 \mathrm{~nm}$ at $\mathrm{P}=30 \mathrm{~mW}$ over $2.5 \mathrm{~mm}$ in $y z$; (h-j) Measured beam width versus $\mathrm{z}$ for various input beam excitations and sizes.

Figure 4 presents the experimental results with two beams, namely a near-infrared pump $(\lambda=1064 \mathrm{~nm})$ and a probe $(\lambda=671 \mathrm{~nm})$. The $1064 \mathrm{~nm}$ beam was launched with a waist $10 \mu \mathrm{m}$ and excited the fundamental mode, as visible in the output profile with a single hump (Fig. 4(a)); the output profile in the red had 4 humps, indicating a multimode waveguide as visible in Fig. 4d. Increasing the pump beam power led to self-focusing of the nearinfrared and a reduction of the corresponding size of the waveguide for the red guided probe. When a nematicon was created at $\mathrm{P}=20 \mathrm{~mW}$ (Fig. 4b) or above (Fig. 4c) the red low power probe became guided into a first order (Fig. 4e) or a higher-order eigenmode (Fig. 4f). We observed the transition of the guided red light from a multi-hump to a single hump by increasing the power of the input pump beam. Further increases of near-infrared nematicon power led to a soliton waveguide supporting more guided modes. The nematicon remained well confined along $\mathrm{y}$ and $x$ with an unchanged size along propagation, but the induced waveguide supported more guided modes of the red probe, as clearly seen in the output cross section (Fig. 4f), where a multi-hump profile indicates a multimode structure. The refractive potential got stronger and wider with increased pump beam power, yielding a multimode channel waveguide for the probe.

In conclusion, in a nonlocal nonlinear material system with non-uniform refractive index distribution, in the linear regime we observed and characterized a multimode

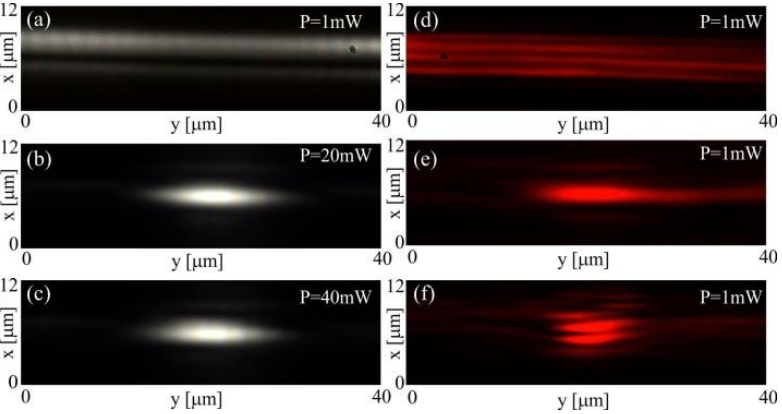

Fig. 4. Experimental results for beams at $1064 \mathrm{~nm}$ (left) and $671 \mathrm{~nm}$ (right) launched collinearly. Output profile of the linear $(\mathrm{P}=1 \mathrm{~mW})(\mathrm{a}) 1^{\text {st }}$ order mode at $1064 \mathrm{~nm}$ and (d) higher order TE eigenmode at $671 \mathrm{~nm}$, respectively; (b) output profile of the nonlinear mode at $1064 \mathrm{~nm}$ for $\mathrm{P}=20 \mathrm{~mW}$ and (e) corresponding guided mode of the probe at $671 \mathrm{~nm}$; (c, f) as in (b, e) but for a near-infrared beam power $\mathrm{P}=40 \mathrm{~mW}$.

structure, with high-order modes propagating along $z$ and diffracting in $y z$. In the nonlinear regime we obtained modal transformation into two dimensional self-confined solitons: the beam became confined in the $y$ plane while propagating as a fundamental nonlinear mode.

The results were confirmed in pump-probe beam experiments using different wavelengths. We showed that a 2D nematicon can support guided modes of a probe signal: from fundamental to higher order modes as the pump excitation increases.

This work was supported by the National Science Centre under the grant agreement DEC2012/06/M/ST2/00479. GA acknowledges support from the Academy of Finland through the FiDiPro grant no. 282858 .

\section{References}

[1] M. Peccianti, A. Dyadyusha, M. Kaczmarek, G. Assanto, Nature Phys. 2, 737 (2006).

[2] G. Assanto, M.A. Karpierz, Liq. Cryst. 36, 1161 (2009).

[3] M. Peccianti, G. Assanto, Phys. Rep. 516, 147 (2012).

[4] A. Piccardi, G. Assanto, L. Lucchetti, F. Simoni, Appl. Phys. Lett. 93 (17), 171104 (2008)

[5] A. Pasquazi, A. Alberucci, M. Peccianti, G. Assanto, Appl. Phys. Lett. 87 (26), 261104 (2005).

[6] M.-F. Shih, M. Segev, G. Salamo, Opt. Lett. 21, 931 (1996).

[7] M. Peccianti, G. Assanto, A. De Luca, C. Umeton, I. C. Khoo, Appl Phys. Lett. 77, 7 (2000).

[8] J. Petter, C. Denz, Opt. Commun. 188, 55 (2001)

[9] M. Peccianti, G. Assanto, Opt. Lett. 26 (21), 1690 (2001)

[10] Y.V. Isdebskaya, A.S. Desyatnikov, G. Assanto, Y. Kivshar, Opt. Lett. 36, 184 (2011).

[11] P. Oswald, P. Pieranski, Nematic and Cholesteric Liquid Crystals (Taylor \& Francis 2005).

[12] A. Laudyn, M. Kwasny, M.A. Karpierz, Appl. Phys. Lett. 94, 091110 (2009).

[13] U.A. Laudyn, P. Jung, K.B. Zegadło, M.A. Karpierz, G. Assanto, Opt. Lett. 39 (22), 6399 (2014).

[14] F. Sala, M.A. Karpierz, Opt. Expr. 20(13), 13923 (2012).

[15] U. Laudyn, P. Jung, M.A. Karpierz, G. Assanto, Sci. Rep. 6, 22923 (2016).

[16] The 1110 nematic mixture was synthesized by Prof. R. Dabrowski at the Military University of Technology in Warsaw 